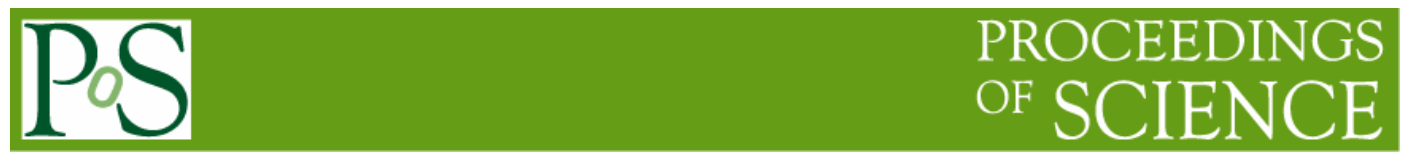

\title{
W \& Z boson production (LHC)
}

\author{
Aristotelis Kyriakis ${ }^{1,2}$ \\ Institute of Nuclear Physics, NCSR 'Demokritos' \\ 15310 Agia Paraskevi, Athens, Greece \\ E-mail: Aristoteles.Kyriakis@cern.ch
}

The measurement of the inclusive $W$ and $Z$ production cross-section using their muon and electron decay modes studied by the ATLAS and CMS collaborations is presented. These studies were performed at both $14 \mathrm{TeV}$ and $10 \mathrm{TeV}$ Centre of Mass Energy and for luminosities $50 \mathrm{pb}^{-1}$ and $10 \mathrm{pb}^{-1}$ respectively. Data driven techniques for the efficiency determination and the background estimation are also discussed. Finally results for the $\mathrm{W}$ charge asymmetry and the $Z$ Boson Forward-Backward asymmetry are also presented

European Physical Society Europhysics Conference on High Energy Physics EPS-HEP 2009, Krakow, Poland July 16-22, 2009

Speaker

On behalf of ATLAS and CMS Collaborations 


\section{Introduction}

Both ATLAS[1] and Compact Muon Solenoid-CMS[2] detectors were designed to explore the nature of the electroweak symmetry breaking and to search for physics beyond the Standard Model. In order for this to be feasible the detectors systematics should be under control. One way to achieve this is to study well understood standard processes like $W$ and $Z$ production.

$W$ and $Z$ bosons are calculated to have large production cross-sections at the LHC[3] and can be easily triggered through their high transverse momenta $\left(P_{t}\right)$ and well-isolated leptonic decay modes. Thus they provide a clean experimental signature with rather low background especially for the $Z$ production.

The analysis strategy for the early data is based on the development of data-driven methods for measuring efficiencies and estimate the signal and background yields. This is because Monte Carlo simulation will not be yet accurate enough and only a preliminary version of the detectors calibration and alignment will be available.

\section{The ATLAS and CMS detectors}

The ATLAS detector consists of an inner tracking system(composed of three subdetectors: Pixel, Silicon microstrip and Transition Radiation Tracker), with pseudo-rapidity coverage of $|\eta|<2.5$, inside a 2 Tesla solenoidal magnetic field, followed by Electromagnetic ( Liquid Argon sampling calorimeter ) and Hadronic( Iron/Scintillator) Calorimeters that cover the pseudo-rapidity region up to $|\eta|<4$.9. In the outer part a muon spectrometer is installed, with pseudo-rapidity coverage of $|\eta|<2.7$, inside a large toroidal magnet system.

The CMS detector consists of an inner tracking system(Pixel and Silicon microstrip) inside a 4 Tesla solenoidal magnetic field, with pseudo-rapidity coverage of $|\eta|<2.5$, followed by an $\mathrm{PbWO}_{4}$ crystal Electromagnetic Calorimeter(ECAL), with pseudo-rapidity coverage of $|\eta|<3.0$ and a Copper/Scintillator Hadronic Calorimeter(HCAL) together with a Forward Hadron Calorimeter(HF), with pseudo-rapidity coverage of $|\eta|<5$.0. Outside the solenoid there are 4 layers of muon spectrometer, inside the solenoid return yoke, while in frond of the Endcap Electromagnetic Calorimeter the Preshower, a silicon-based detector for photon - neutral pion separation is installed.

\section{W and $\mathrm{Z}$ selection}

ATLAS based the $W \rightarrow e v_{e}\left(Z \rightarrow e^{+} e^{-}\right)$analysis on a single electron trigger with $P_{t}>20 \mathrm{GeV}$ $/ \mathrm{c}\left(P_{t}>10 \mathrm{GeV} / \mathrm{c}\right)$ for pre-selection and then requiring one (two) EM cluster(s) with $E_{t}>25 \mathrm{GeV}$ $\left(E_{t}>15 \mathrm{GeV}\right)$ and $|\eta|<2.4$. Electron identification criteria based on tracker and EM cluster shape information were also applied followed by isolation of the EM clusters in the Electromagnetic Calorimeter. The $\mathrm{e}^{+} \mathrm{e}^{-}$invariant mass distribution for the signal and the major jet background for $50 \mathrm{pb}^{-1}$ luminosity and $14 \mathrm{TeV}$ centre of mass energy is shown in figure 1 . The $W$ analysis includes also cuts in the reconstructed missing transverse energy, reflecting the missing final state neutrino $\left(E_{t}^{\text {miss }}>25 \mathrm{GeV}\right)$ and the transverse mass of the $(l, v)$ system $\left(M_{T}>\right.$ $\left.40 \mathrm{GeV} / \mathrm{c}^{2}\right)$. Similarly the $W \rightarrow \mu v_{\mu}\left(Z \rightarrow \mu^{+} \mu^{-}\right)$analysis based on single muon trigger with $P_{t}>20$ $\mathrm{GeV} / \mathrm{c}\left(P_{t}>10 \mathrm{GeV} / \mathrm{c}\right)$ for pre-selection and the requirement of one (two opposite sign) muon 
$\operatorname{track}(\mathrm{s})$ with $P_{t}>25 \mathrm{GeV} / \mathrm{c}\left(P_{t}>20 \mathrm{GeV} / \mathrm{c}\right)$ and $|\eta|<2.5$ while for each muon isolation criteria from tracker were also applied. Same conditions for $E_{t}^{\text {miss }}$ and $M_{T}$ as in the electron case. In figure 2 the $M_{T}$ for the $W \rightarrow \mu v_{\mu}$ signal and background and for $50 \mathrm{pb}^{-1}$ luminosity and $14 \mathrm{TeV}$ centre of mass energy is shown[4].

CMS selected the $W \rightarrow e v_{e}\left(Z \rightarrow e^{+} e^{-}\right)$sample using single electron trigger and then requiring one (two) electrons (formed as EM clusters associated with tracks) with $E_{t}>30 \mathrm{GeV}\left(E_{t}>20 \mathrm{GeV}\right)$ and $|\eta|<2.5$ (excluding the ECAL Barrel-Endcap region). The electrons are also required to satisfy electron identification criteria based on shower shape variables like the width of the EM cluster in $\eta$ direction and the quality of the EM cluster-tracker matching. The electrons should be isolated as well, by requiring low charged and/or neutral particle activity in a cone around the electron candidate direction. The Missing Energy distribution $\left(E_{t}^{\text {miss }}\right)$ for the $W \rightarrow e v_{e}$ signal and the major backgrounds after all cuts for $10 \mathrm{pb}^{-1}$ luminosity and $10 \mathrm{TeV}$ centre of mass is shown in figure 3[5]. Similarly the $W \rightarrow \mu v_{\mu}\left(Z \rightarrow \mu^{+} \mu^{-}\right)$sample was selected using single muon trigger and then requiring one (two opposite sign) muons with $P_{t}>25 \mathrm{GeV} / \mathrm{c}\left(P_{t}>20 \mathrm{GeV} / \mathrm{c}\right)$ and $|\eta|<2.1$. The muons should be isolated by requiring low charge particle activity in a cone around the muon candidate direction[6]. The $\mu^{+} \mu^{-}$invariant mass distribution for the signal and the major jet background for $10 \mathrm{pb}^{-1}$ luminosity and $10 \mathrm{TeV}$ centre of mass energy is shown in figure 4.

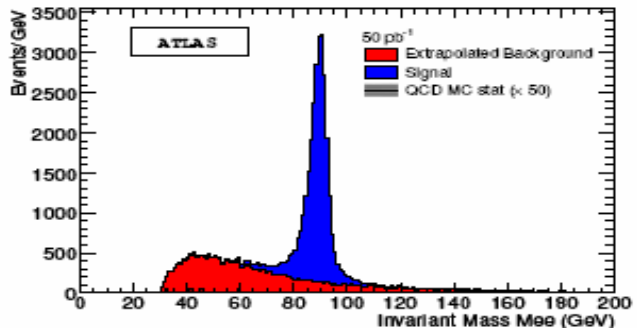

Figure 1: The e $e^{+} e^{-}$invariant mass distribution for signal and the major backgrounds after all cuts for $50 \mathrm{pb}^{-1}$ luminosity and $14 \mathrm{TeV}$ centre of mass energy

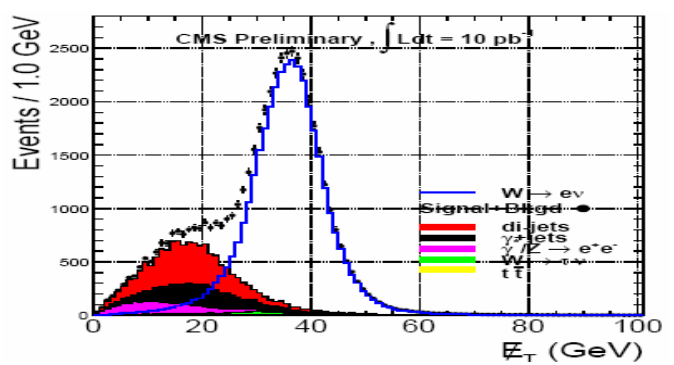

Figure 3: The Transverse Missing Energy $\left(E_{t}^{\text {miss }}\right)$ distribution for the $W \rightarrow e v_{e}$ signal and the major backgrounds after all cuts for $10 \mathrm{pb}^{-1}$ luminosity and $10 \mathrm{TeV}$ centre of mass energv

\section{Data-driven methods for efficiency determination and background estimation}

In order to calculate from data the efficiencies of the various selection criteria, described in section 3 for the cross-section measurement of the $W$ and $Z$ production, the 
Tag and Probe $(T \& P)$ method[7] was used. This method relies on the $Z \rightarrow \mu^{+} \mu^{-}\left(e^{+} e^{-}\right)$ decays that provide a pure and unbiased sample of muons(electrons). One of the muon(electron), so called tag, should satisfy very stringent identification criteria whilst the other lepton, so called probe, should have an invariant mass together with the tag lepton close to the $M_{Z}$ (to ensure the purity of the probe sample) and should satisfy a subset of selection criteria depending on the efficiency under study. The efficiencies measured with the method have been validated against the true efficiencies from MC simulations(Figure 5).

The EW backgrounds in $W$ and $Z$ production are quite small. Taking into account that their cross-sections are also well understood theoretically, makes their estimation from $\mathrm{MC}$ simulation reliable. On the contrary the $Q C D$ background especially in case of $W \rightarrow e v_{e}$ is significant and hard to be estimated from simulation and has to be measured from data. Once efficiencies are known from data using the $T \& P$ method, the signal and background yields can be solved algebraically in terms of the total observed event yield above and below a transverse missing energy ( $E_{t}^{\text {miss }}$ ) requirement(Figure 3 ). The question is to find a way to reconstruct the missing transverse energy from data. The answer is given by the template method in which the $\mathrm{Z} \rightarrow \mathrm{e}^{+} \mathrm{e}^{-}$sample used to calculated the $E_{t}^{\text {miss }}$ after removing one of the electrons and correcting for the different kinematics between the $W \rightarrow e v_{e}$ and $Z \rightarrow e^{+} e^{-}$events. Figure 6 shows the comparison between the ersatz $E_{t}^{\text {miss }}$ (red line) as calculated from the $Z \rightarrow e^{+} e^{-}$sample and the $E_{t}^{\text {miss }}$ as calculated from the $W \rightarrow e v_{e}$ events (blue dashed line). Thus the ersatz $E_{t}^{m i s s}$ is a reasonable representation of the true $E_{t}^{\text {miss }}$ from the $W \rightarrow e v_{e}$ events.

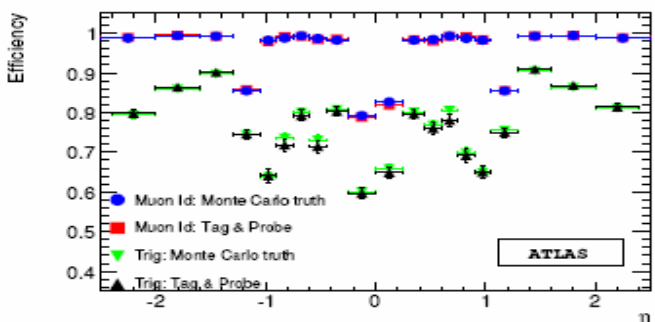

Figure 5: Muon detection efficiency vs $\eta$, as measured by the $T \& P$ method and compared to the truth for $50 \mathrm{pb}^{-1}$

\section{Cross-section Measurement}

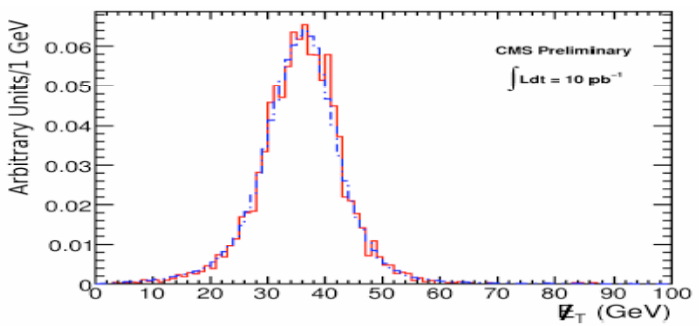

Figure 6: Comparison between the ersatz $E_{t}^{\text {miss }}$ (red line) as calculated from the $Z \rightarrow e^{+} e^{-}$sample and the $E_{t}^{\text {miss }}$ as calculated from the $W \rightarrow e v_{e}$ events (blue dashed line).

The $W$ and $Z$ production cross-section is calculated using the following formula:

$$
\sigma \times \mathrm{B} r(Z / W \rightarrow \text { leptons })=\frac{N-B}{L \times A \times \varepsilon}
$$

where $N$ and $B$ are the number of signal and background events respectively that passed the selection criteria, $\varepsilon$ is the trigger, reconstruction and selection efficiency measured from data using the T\&P method described in section $4, A$ is the geometric and kinematic acceptance determined from $\mathrm{MC}$ simulation and $L$ is the integrated luminosity measured in an independent way. Analysis showed that ATLAS has a systematic uncertainty of $3.1 \%(3.8 \%)$ for the $W \rightarrow \mu v_{\mu}\left(Z \rightarrow \mu^{+} \mu^{-}\right)$channel at $50 \mathrm{pb}^{-1}$ and $14 \mathrm{TeV}$ centre of mass energy, whilst CMS has a systematic uncertainty of $4.0 \%(2.4 \%)$ for the $W \rightarrow e v_{e}\left(Z \rightarrow e^{+} e^{-}\right)$channel at $10 \mathrm{pb}^{-1}$ and $10 \mathrm{TeV}$ centre of mass energy. An extra $10 \%$ uncertainty should be also taken into account for the luminosity. 


\section{Muon charge asymmetry}

The muon charge asymmetry $\mathrm{A}(\eta)$ is defined as follows:

$$
A(\eta)=\frac{\frac{d \sigma}{d \eta}\left(W^{+} \rightarrow \mu^{+} v\right)-\frac{d \sigma}{d \eta}\left(W^{-} \rightarrow \mu^{-} v\right)}{\frac{d \sigma}{d \eta}\left(W^{+} \rightarrow \mu^{+} v\right)+\frac{d \sigma}{d \eta}\left(W^{-} \rightarrow \mu^{-} v\right)}
$$

and is rather insensitive to systematic uncertainties coming from misalignment and/or mis-calibration. This measurement allows probing the parton density functions $(P D F s)$ of the incoming $u$ and $d$ quarks. The $W \rightarrow \mu v_{\mu}$ selection criteria are the same as those described in the cross-section measurement and the analysis performed by the CMS collaboration showed that $A(\eta)$ can constrain current $P D F$ s sets with $100 \mathrm{pb}^{-1}$ at $10 \mathrm{TeV}$ centre of mass energy[8].

\section{Z Boson Forward - Backward asymmetry $\left(A_{F B}\right)$}

In $p p$ collisions the $e^{+} e^{-}$pairs are predominantly produced via quark anti-quark annihilation. In Standard Model there is an asymmetry $\left(A_{F B}\right)$ in the polar emission angle $(\theta)$ of the electron relatively to the quark momentum vector in the $e^{+} e^{-}$rest frame. The $A_{F B}$ measurement can improve the knowledge of the weak mixing angle $\sin ^{2} \theta^{\text {eff }}$ lept since it is expected a linear dependence between these two quantities. The $A_{F B}$ can be given by the formula: $A_{F B}=\left(N_{F}-N_{B}\right) /\left(N_{F}+N_{B}\right)$ where $N_{F}\left(N_{B}\right)$ represents the number of forward (backward) produced events. ATLAS performed an analysis on $A_{F B}$ exploiting the capability to reconstruct electrons up to large $\eta$ regions (up to $|\eta|<4$.9) supposing luminosity $100 \mathrm{fb}^{-1}$ at $14 \mathrm{TeV}$ centre of mass energy. The estimated error in the $\sin ^{2} \theta_{\text {eff }}^{\text {lept }}$ measurement is : $(1.5($ stat $) \pm 0.3(\exp ) \pm 2.4(P D F)) \times 10^{-4}[4]$.

\section{Conclusions}

Inclusive $W / Z$ cross-section measurements studies have been performed in both ATLAS and CMS experiments at LHC. Data-driven methods were tested for extracting efficiencies and $Q C D$ background. All these, together with muon charge asymmetry and $Z$ boson forward-backward asymmetry studies represent a robust start-up program. The control of the SM Electroweak processes gives also a significant confidence for New Physics studies.

\section{References}

[1] ATLAS Collaboration,The ATLAS Experiment at the CERN LHC,JINST 3:S08003,2008.

[2] CMS Collaboration, The CMS Experiment at the CERN LHC,JINST 3:S08004,2008.

[3] Standard Model Physics (and more) at the LHC,CERN-2000-004.

[4] ATLAS Collaboration, Expected Performance of the ATLAS Experiment, CERN-OPEN-2008-020

[5] CMS Collaboration, CMS PAS EWK-09-004

[6] CMS Collaboration, CMS PAS EWK-09-001

[7] CMS Collaboration, Measuring Electron Efficiencies at CMS with Early Data,CMS PAS EGM2007/001.

[8] CMS Collaboration, CMS PAS EWK-09-003 